\title{
Radioprotective effect of a pan-caspase inhibitor in a novel model of radiation injury to the nucleus of the abducens nerve
}

\author{
JIANGUO LI ${ }^{1,2 *}$, LI LIN $^{3 *}$, LIQING DU ${ }^{2}$, CHANG XU ${ }^{2}$, YAN WANG $^{2}$, JIA CAO $^{2}$, QIN WANG $^{2}$, \\ FEIYUE FAN ${ }^{2}$, XIAOPING WANG ${ }^{1}$, YAFEI WANG ${ }^{3}$ and QIANG LIU ${ }^{2}$ \\ ${ }^{1}$ Department of Human Anatomy, The Medical School of Inner Mongolia University for the Nationalities, Neimenggu, \\ Tongliao 028041; ${ }^{2}$ Tianjin Key Laboratory of Molecular Nuclear Medicine, Institute of Radiation Medicine, \\ Chinese Academy of Medical Sciences and Peking Union Medical College, Tianjin 300192; \\ ${ }^{3}$ Key Laboratory of Cancer Prevention and Therapy, Department of Hematology, \\ Tianjin Medical University Cancer Institute and Hospital, Tianjin, 300060, P.R. China
}

Received October 18, 2013; Accepted May 19, 2014

DOI: $10.3892 / \mathrm{mmr} .2014 .2334$

\begin{abstract}
There is increasing evidence that neuronal cell death occurs via extrinsic (death receptors) and intrinsic (mitochondria) pathways. Radiation induces caspase activation fundamentally via the mitochondrial pathway. Caspases are the key regulators of apoptosis. Healthy male Sprague-Dawley rats were used in the present study to examine the radioprotective effect of a type of pan-caspase inhibitor, z-VAD-fmk, following radiation, to investigate the effects of caspase blockade in a model of the nucleus of the abducens nerve. z-VAD-fmk was injected intracerebroventricularly as a bolus injection $(0.2 \mu \mathrm{g} / \mathrm{h}$ for $1 \mathrm{~h}$ ) into rats prior to exposure to radiation. Irradiation was conducted at room temperature at a dose of radiation of $4 \mathrm{~Gy}$. The present study performed immunohistochemistry, terminal deoxynucleotidyl transferase dUTP nick end labeling (TUNEL) and western blot analysis and identified no significant changes in the expression of the X-linked inhibitor of apoptosis protein (XIAP) following radiation $(\mathrm{P}>0.05)$. As compared with the radiation alone group, the quantification of TUNEL-positive neurons was reduced in $\mathrm{z}$-VAD-fmk-treated animals following radiation $(\mathrm{P}<0.01)$. Inhibition of caspase induced by $\mathrm{z}-\mathrm{VAD}-\mathrm{fmk}$
\end{abstract}

Correspondence to: Dr Qiang Liu, Tianjin Key Laboratory of Molecular Nuclear Medicine, Institute of Radiation Medicine, Chinese Academy of Medical Sciences and Peking Union Medical College, 238 Baidi Road, Nankai, Tianjin 300192, P.R. China E-mail: qiangliu70@yahoo.com

Dr Yafei Wang, Key Laboratory of Cancer Prevention and Therapy, Department of Hematology, Tianjin Medical University Cancer Institute and Hospital, Huanhu Xi Road, Hexi, Tianjin, 300060, P.R. China

E-mail: wangyafeitj@126.com

*Contributed equally

Key words: radiation, brain stem, caspase, caspase inhibitors, apoptosis reduced the expression and activation of caspase-3, -8 and -9 $(\mathrm{P}<0.01)$. z-VAD-fmk effectively prevented radiation-induced apoptosis and this caspase inhibitor may be a potential therapeutic target in the treatment of brain radiation injury. The nucleus of the abducens nerve may be used as a radiation injury model, providing visual information and data on the apoptotic morphology of the abducens nucleus.

\section{Introduction}

Neuronal cell apoptosis is associated with various neurological damaging factors, including radiation (1). Studies on the molecular mechanism of neuronal cell apoptosis following radiation have enriched the number of protective therapeutic strategies against radiation-induced neuronal cell death (2). Unlike inflammation, apoptosis is a 'programmed cell death' mechanism, whereby enzymatic reactions lead to cell apoptosis and phagocytes remove the debris without stimulating an inflammatory response. The most important enzymes involved in apoptosis are caspases, which hydrolyze important structural and functional proteins of the cell, ultimately leading to apoptosis. Caspases are synthesized in the cell as inactive zymogens and require to be activated to be functional. There are two pathways that stimulate the activation of caspases; the extrinsic and intrinsic cascades $(3,4)$. The extrinsic pathway is associated with membrane receptors and their ligands, and the intrinsic pathway is dependent on mitochondria (5). The extrinsic pathway is triggered by binding of death ligands, such as tumor necrosis factor (TNF)- $\alpha$, to death receptors of the TNF family, which results in the assembly of a receptor-associated complex. The central element in the mitochondrial pathway is a specialised protein complex, the apoptosome, which enables and facilitates the activation of procaspase 9. Once activated by their respective upstream signals, caspase- 8 and -9 may cleave and activate downstream executioner caspases -3 and -7 , which, in turn, cleave a plethora of target proteins, resulting in apoptotic death (6-8).

Radiation and other agents induce caspase activation fundamentally via the mitochondrial pathway, which includes mitochondrial integration of apoptotic signals and the 
subsequent release of cytochrome $\mathrm{c}$ into the cytosol $(5,9,10)$. The inhibitor of apoptosis proteins (IAPs) inhibit apoptosis by interacting with and then regulating the functions of caspase- 8 or caspase-9, -3 and $-7 \quad(9,11,12)$. z-VAD-fmk ( $N$-benzyloxycarbonyl-Val-Ala-Asp-fluoromethylketone) is a powerful, irreversible and cell permeable inhibitor of caspases, and has been demonstrated to directly block the activity of caspases (13).

As a result, the present study aimed to investigate changes in the expression of X-linked IAP (XIAP) induced by radiation injury, the activity and expression of caspase members following radiation and the effect of caspase blockade. In the present study, a model of the nucleus of abducens nerve was established to examine this.

\section{Materials and methods}

Radiation mode. The rats were housed in groups of four to five per cage at a temperature- $\left(24 \pm 1^{\circ} \mathrm{C}\right)$ and light-controlled room (12 h light/dark cycle with lights on at 07:00 h). Food and water were provided ad libitum before and after treatment. The animal care and all experimental procedures were carried out in accordance with the Guide for the Care and Use of Laboratory Animals published by the US National Institutes of Health (publication no. 85-23, revised 1996). Male Sprague-Dawley rats weighing 200-220 g were obtained from the Experimental Animal Research Center, Institute of Radiation Medicine, Chinese Academy of Medical Sciences and Peking Union Medical College (Tianjin, P.R. China) and randomly divided into three groups (six rats/group): the irradiation group (IR group), the irradiation with $\mathrm{z}$-VAD-fmk group (IR + Z-VAD group) and the control group (con group). Irradiation was performed at room temperature at a dose of radiation (4 Gy) with a $\mathrm{Cr} 137$ r-ray (Atomic Energy of Canada Ltd., Mississauga, ON, Canada) at a dose rate of $0.71 \mathrm{~Gy} / \mathrm{min}$. The animals in the control group did not receive any radiation.

Intracerebroventricular administration of z-VAD-fmk. With a rat brain stereotaxic apparatus (Stoelting Co., Wood Dale, IL, USA), the animals were implanted with a cannula ( $\mathrm{AP}=-2.4 \mathrm{~mm}$, $\mathrm{L}=-1.4 \mathrm{~mm}, \mathrm{H}=-3.0 \mathrm{~mm}$ ) intracerebroventricularly (i.c.v.) via an osmotic micropump (Alzet ${ }^{\circledR}$ micropump, 1007D; Durect Corporation, Cupertino, CA, USA). Infusion of $2 \mu \mathrm{g} \mathrm{z-VAD-fmk}$ (BioVision, Inc., Milpitas, CA, USA) in $10 \mu \mathrm{l}$ volume was conducted at a rate of $0.2 \mu \mathrm{g} / \mathrm{h}$ for $1 \mathrm{~h}$. The drug vehicle was $0.5 \%$ dimethyl sulfoxide (DMSO) in phosphate-buffered saline (PBS). The infusions were performed at the onset of radiation administration (13). The non-radiation controls received PBS and vehicle i.c.v. and the radiation controls received z-VAD-fmk. The animals were sacrificed $24 \mathrm{~h}$ following administration of diazepam for further investigations.

Immunohistology and terminal deoxynucleotidyl transferase dUTP nick end labeling (TUNEL) staining. Brains were harvested and immediately frozen in 2-methylbutane $\left(-30^{\circ} \mathrm{C}\right)$. The brainstem was cut into sections (12- $\mu \mathrm{m}$ thick) with a Leica CM 3000 cryostat (Leica Microsystems, Wetzlar, Germany) at the level of the nucleus of the abducens nerve (14), and then stored at $-80^{\circ} \mathrm{C}$ until further use. Coronal sections were air dried for $15 \mathrm{~min}$, post-fixed in $10 \%$ formalin for $15 \mathrm{~min}$, washed twice in PBS and then processed for immunohistology with rabbit anti-XIAP (1:1,500 dilution; Abcam, Cambridge, MA, USA). The avidin-biotin-peroxidase complex method was conducted as previously described $(15,16)$. For detection of DNA fragmentation, the fluorescein-based TUNEL assay (Roche Molecular Biochemicals, Indianapolis, IN, USA) was used. TUNEL staining was conducted according to the manufacturer's instructions. TUNEL cell counts were performed on $n=3$ brain sections from the nuclei of the abducens nerves. Images were visualized using a Leica microscope (DMI3000B; Leica Microsystems, Wetzlar, Germany) under excitation/emission wavelengths of 500/550 nm (green), captured using an Optronics DEI-750 three-chip camera equipped with a BQ 8000 sVGA frame grabber and analyzed using Bioquant software (Bioquant Image Analysis Corporation, Nashville, TN, USA).

Generation of cytosolic fraction. Twenty-four hours subsequent to irradiation, the rats from each group were anesthetized with $10 \%$ chloral hydrate ( $30 \mathrm{mg} / \mathrm{kg}$ body weight) by intraperitoneal anesthesia and the brainstems containing the nuclei of the abducens nerves were obtained. The cytosolic fraction was performed as previously described (16).

Western blot analysis. The protein concentration of the supernatant homogenate was determined using a Bio-Rad kit (Bio-Rad, Hercules, CA, USA) at an absorbance of $595 \mathrm{~nm}$ with the Bradford method (17). The samples $(80 \mu \mathrm{g})$ were transferred to polyvinylidene difluoride membranes and incubated with the following primary antibodies: Rabbit polyclonal anti-XIAP from Abcam (dilution, 1:500), rabbit anti- $\beta$-actin (dilution, 1:1,500; Sangon Biotech, Shanghai, China) and goat anti-rabbit immunoglubulin G-conjugated to horseradish peroxidase (dilution, 1:800; ZSGB Biotechnology, Co., Ltd., Beijing, China).

RNA extraction, $c D N A$ synthesis and quantitative polymerase chain reaction $(q P C R)$. Total RNA was purified and extracted as conducted previously by our laboratory (18). The expression of a target gene was calculated by the comparative CT method [fold-change $\left.=2^{(-\Delta \Delta C T)}\right]$. The PCR primers for caspase-3, -8 and -9 as well as the housekeeping gene GAPDH were obtained from Sangon Biotech. The specific primer pairs used were: CASP3, 5'-ATCACAGCAAAAGGAGCAGTTT-3' (forward) and 5'-ACACCACTGTCTGTCTCAATGC-3' (reverse); CASP8, 5'-TAGGGACAGGAATGGAACACA-3' (forward) and 5'-TGGGAGAGGATACAGCAGATG-3' (reverse); CASP9, 5'-TCTGGAGGATTTGGTGATGTC-3' (forward) and 5'-CATTTTCTTGGCAGTCAGGTC-3' (reverse); GAPDH, 5'-ATGACATCAAGAAGGTGGTG-3' (forward) and 5'-CATACCAGGAAATGAGCTTG-3' (reverse).

Caspase activation assay. The activity of caspase-3, -8 and -9, was analyzed using a fluorogenic caspase assay with Ac-DEVDamido-trifluoromethylcoumarin (AFC), Ac-IETD-AFC, Ac-LEHD-AFC (BD Pharmingen) as the substrate, respectively. The results are expressed as the fold-change compared with the control as previously described (18).

Statistical analysis. Data are expressed as the mean \pm standard deviation and analyzed using one-way analysis of variance 

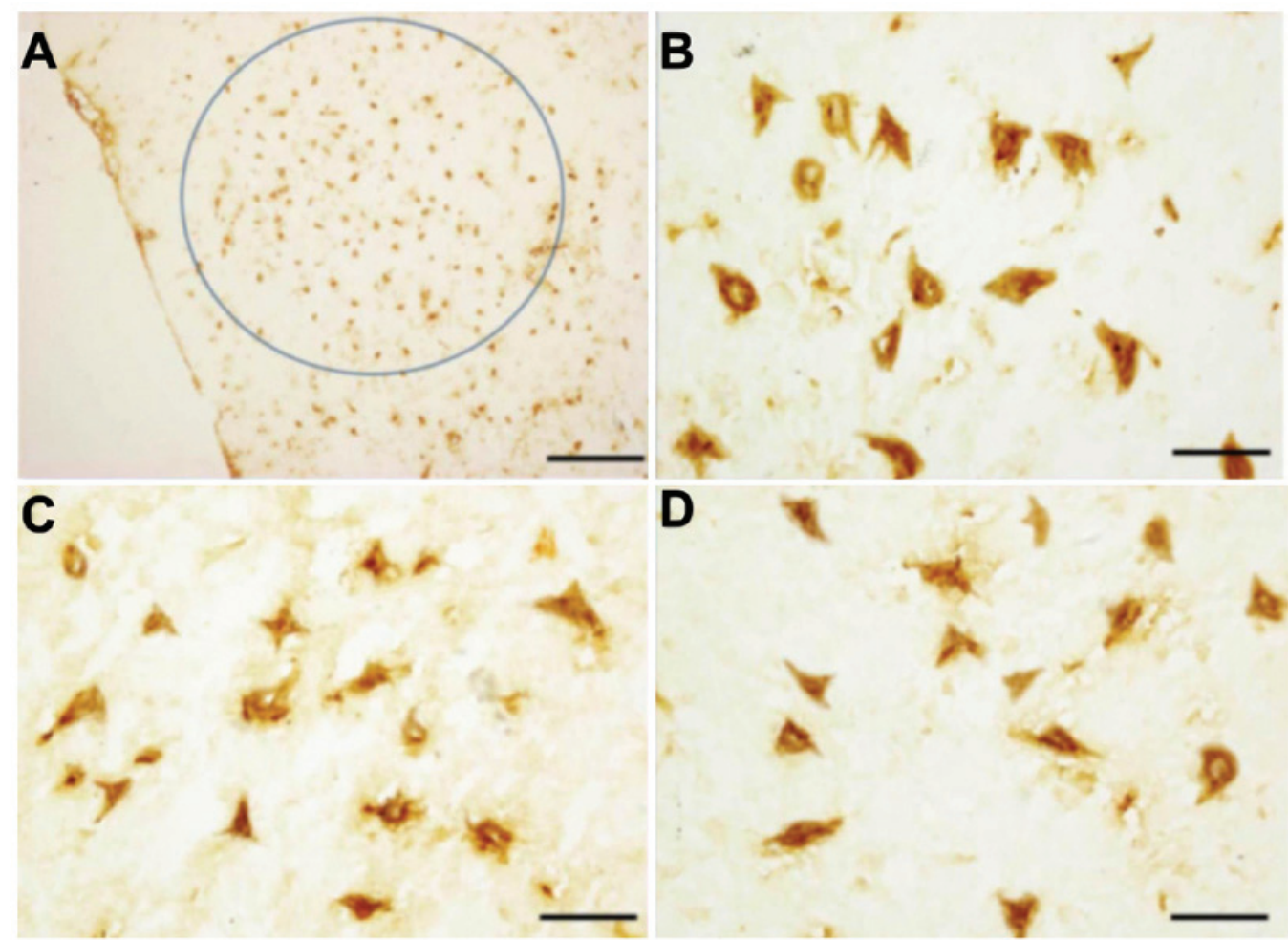

Figure 1. Quantification of XIAP protein expressing cells in nucleus of abducent nerve. XIAP expressed in neurons of the abducens nucleus from (A and B) control rats, (C) radiation rats and (D) z-VAD-fmk-treated rats. (A) Circle denotes the location of nucleus of the abducens nerve. Scale bars: (A), $100 \mu \mathrm{m}$; (B-D), $20 \mu \mathrm{m}$. XIAP, X-linked inhibitor of apoptosis protein; z-VAD-fmk, $N$-benzyloxycarbonyl-Val-Ala-Asp-fluoromethylketone.
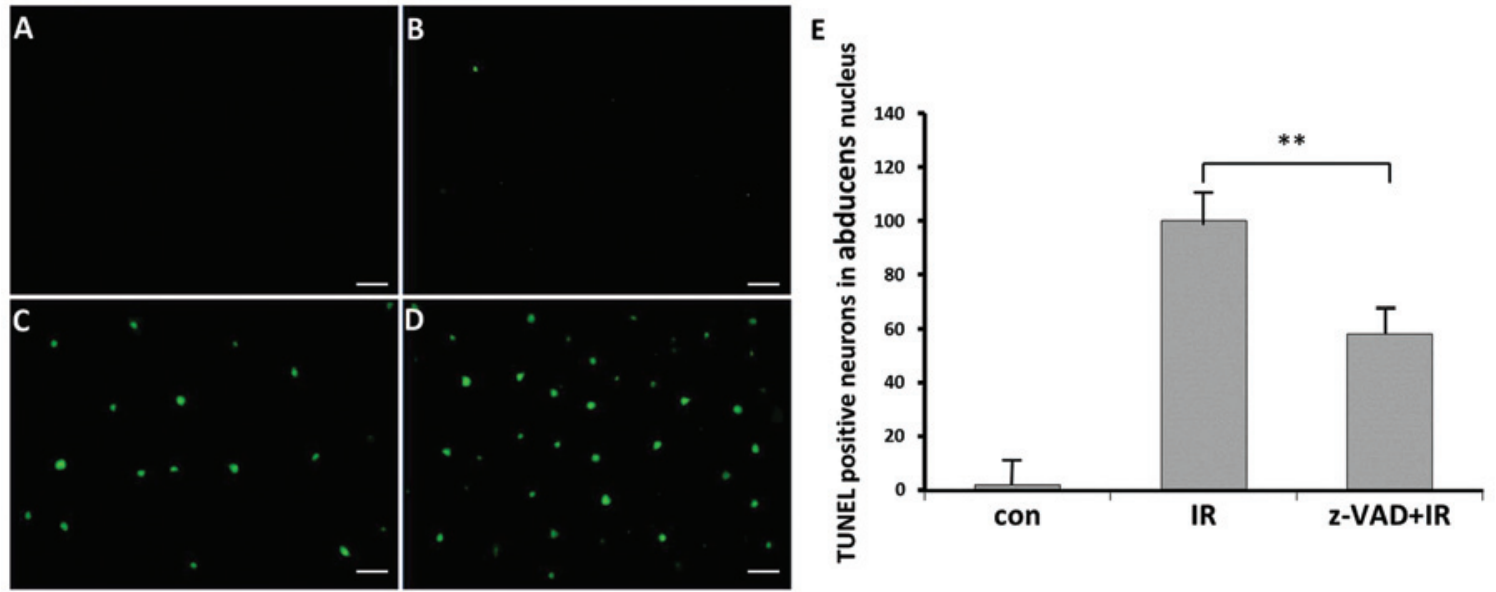

Figure 2. TUNEL-positive neurons in the abducens nucleus. (A) Negative control; (B) control rats; (C) z-VAD-fmk-treated rats and (D) radiation rats. (E) The bar chart presents the count of TUNEL-positive cells. $\mathrm{n}=6$ /group. Scale bar, $80 \mu \mathrm{m}$. Data are expressed as the mean \pm standard deviation. ${ }^{* *} \mathrm{P}<0.01 \mathrm{vs.} \mathrm{radiation}$ group. z-VAD-fmk, $N$-benzyloxycarbonyl-Val-Ala-Asp-fluoromethylketone; IR, irradiation; TUNEL, terminal deoxynucleotidyl transferase dUTP nick end labeling.

with a post-hoc test (multiple comparison test), which was used to determine the significance of the differences between the groups. $\mathrm{P}<0.05$ was considered to indicate a statistically significant difference.

\section{Results}

Expression of XIAP and TUNEL-positive cells within the nuclei of abducens nerves. XIAP was predominantly expressed in the cytoplasm as indicated by positive yellow-brown staining, at high magnification of the brown granulate (Fig. 1). In the normal brain, XIAP was predominantly expressed in the perinuclear region of neurons (Fig. 1A and B). Similar levels of XIAP were present in the brainstems following radiation (Fig. 1C and D). TUNEL-positive cells appeared mainly in the nuclei of abducens nerves of the radiation groups IR and IR+z-VAD (Fig. 2C and D). By contrast, few TUNEL-positive cells were detected in the control rats (Fig. 2B).

Western blot analysis of XIAP following radiation. There was no difference in XIAP expression in the groups of radiated rats, $\mathrm{z}$-VAD-fmk-treated rats and vehicle-treated rats following 
A
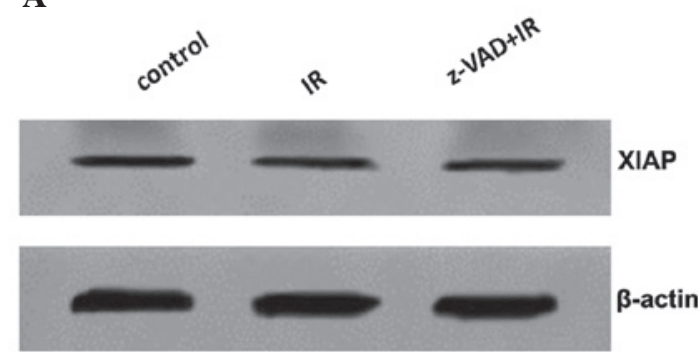

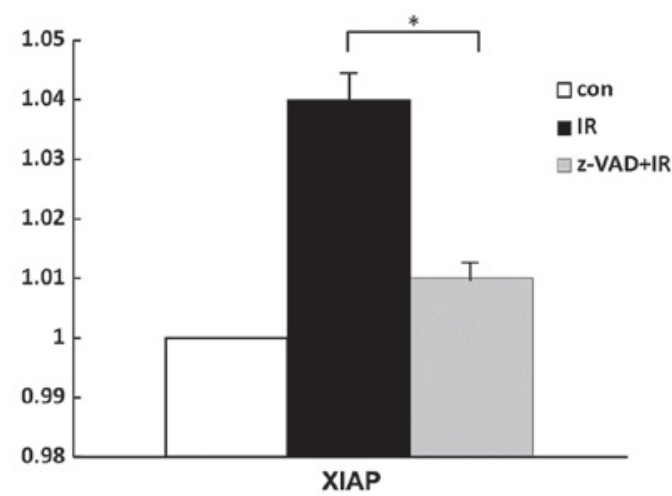

Figure 3. Effect of z-VAD-fmk on the expression of XIAP. (A) Western blot analysis representative pattern from six rats. (B) Histogram data are expressed as the mean \pm standard deviation from six rats of three independent experiments. ${ }^{*} \mathrm{P}>0.05$ vs. radiation group. XIAP, X-linked inhibitor of apoptosis protein; z-VAD-fmk, $N$-benzyloxycarbonyl-Val-Ala-Asp-fluoromethylketone; IR, irradiation.

A

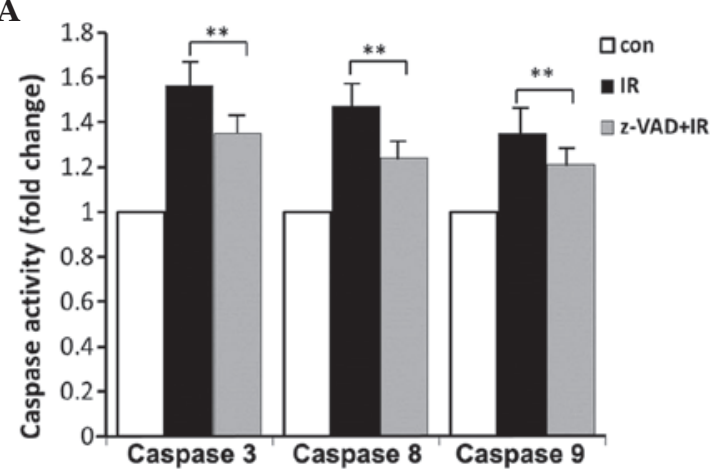

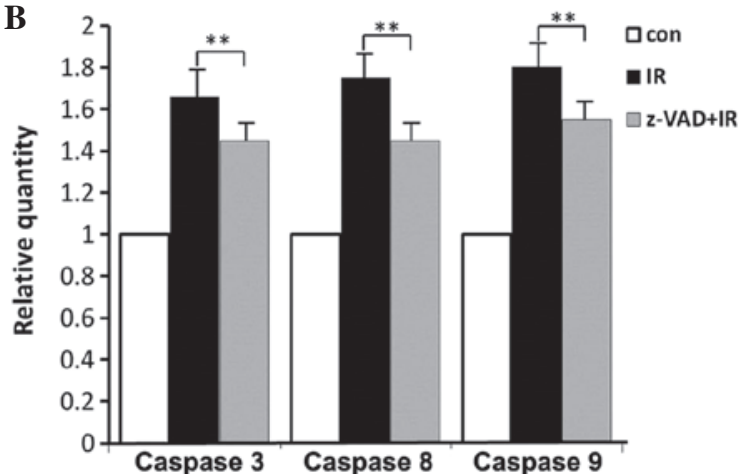

Figure 4. mRNA expression and the activity of caspase-3, -8 and -9 were measured. ${ }^{* *} \mathrm{P}<0.01$ vs. radiation group. Con, control; IR, irradiation; $\mathrm{z}-\mathrm{VAD}-\mathrm{fmk}$, $\mathrm{N}$-benzyloxycarbonyl-Val-Ala-Asp-fluoromethylketone.

radiation (Fig. 3B-D). No significant change was identified in the expression of XIAP following radiation ( $\mathrm{P}>0.05$; Fig. 3 ).

Neuroprotective effects of the pan-caspase inhibitor $z$-VAD-fmk in vivo. Compared with the radiation alone group, the number of TUNEL-positive neurons was reduced in the z-VAD-fmk-treated animals following radiation $(\mathrm{P}<0.01$; Fig. 2E).

Caspase expression and activity. The mRNA expression of caspase- $3,-8$ and -9 was measured. In the brainstem, radiation alone treatment increased the mRNA expression of caspase-3, -8 or -9 by $1.65-, 1.75$ - and 1.80 -fold and enhanced their activity by $1.56-, 1.47-$ and 1.35 -fold, respectively. Combined treatment caused a significant decrease in the mRNA expression of caspase-3, -8 and -9 by $1.45-, 1.45-$ and 1.55 -fold and reduced their activity by $1.35-, 1.24$ - and 1.21 -fold, respectively (Fig. 4A and B).

\section{Discussion}

To completely elucidate the role of caspase in the radiation injury model of the abducens nerve, i.c.v. injection of SpragueDawley rats with z-VAD-fmk, a cell-permeable pan-caspase inhibitor, was performed. z-VAD-fmk reduced the number of TUNEL-positive cells within the nucleus of the abducens nerve. The results demonstrated that inhibition of caspase induced by z-VAD-fmk reduced the expression and activation of caspase- $3,-8$ and -9 , indicating that intervention in the caspase cascade may have applications as a potential protective treatment of brain radiation injury and may represent a therapeutic target.

Evidence has demonstrated that IAP family members are involved in the regulation of caspase activation (19). IAPs inhibit apoptosis by interacting with and then controlling the functions of caspase-8 or caspase-9, -3 and -7 (9). Cellular (c)-IAP1, c-IAP2 and XIAP are three significant members of the IAP family, particularly XIAP, which has numerous domains interacting with different caspases, including caspase-3, -7 and $-9(20,21)$ and its BIR2 domain inhibits caspase-7 in a non-competitive manner (22). XIAP blocks apoptosis at the effector phase, a point where multiple signaling pathways converge $(23,24)$. The majority of the current studies of XIAP have concentrated on its role in cancer or cerebral ischemia reperfusion injury $(11,19)$. By contrast, the effect of XIAP following brain injury induced by radiation remains elusive. In the present study, no significant change was detected in the expression of XIAP when compared with the control. These results indicated that XIAP did not have an important role as an antiapoptotic agent following irradiation.

The nucleus of the abducens nerve has a relatively large volume in the brainstem, and the distribution of neurons is predominantly balanced (25); therefore, locating the nucleus is comparatively simple (Fig. 1A). Since z-VAD-fmk does 
not penetrate the blood-brain barrier (13), it was applied intracerebroventricularly as a bolus injection to overcome this limitation. The injection was administered into the cerebrospinal fluid circulating through the fourth ventricle, allowing z-VAD-fmk to permeate to the neurons through the process of osmosis. This may act as a useful model of radiation injury, providing visual information on the morphology of the apoptotic nucleus. The abducens nucleus contains a large number of mitochondria (26), which was highly useful for the assays conducted in the present study. Changes in the abducens nuleus in an animal model established by exposure to radiation were examined. To the best of our knowledge, the use of this method to study radiation damage and protection has not been reported previously. It is important to note that the suspension was extracted from the cells of the brainstem corresponding to the nucleus of the abducens nerve section. Further studies are required to investigate the effects of radiation in other nuclei, for which novel models will be developed.

In conclusion, z-VAD-fmk effectively prevented radiation-induced apoptosis, and the caspase cascade may be a potential therapeutic target in the treatment of brain radiation injury. The nucleus of the abducens nerve suitable as a radiation injury model, providing visual information and data on the apoptotic morphology of nuclei.

\section{Acknowledgements}

The present study was supported by the Special Foundation of the Ministry of Health (no. 201002009), the National Natural Science Foundation of China (nos. 31170804, 31240052 and 31200634), the Natural Science Foundation of Tianjin (nos. 13JCYBJC23500, 13JCQNJC11600, 11ZCGYSY02400, 12JCYBJC15300 and 12JCYBJC32900) and the PUMC Youth Fund and Fundamental Research Funds for the Central Universities (no. 2012G01,2012J05).

\section{References}

1. Bladen CL, Kozlowski DJ and Dynan WS: Effects of low-dose ionizing radiation and menadione, an inducer of oxidative stress, alone and in combination in a vertebrate embryo model. Radiat Res 178: 499-503, 2012.

2. Loftis GK, Collins S and McDowell M: Anesthesia-induced neuronal apoptosis during synaptogenesis: a review of the literature. AANA J 80: 291-298, 2012.

3. Lisi S, Sisto M, Lofrumento D, Frassanito MA, Caprio S, Romano ML, Mitolo V and D'Amore M: Regulation of mRNA caspase- 8 levels by anti-nuclear autoantibodies. Clin Exp Med 10: 199-203, 2010.

4. Yao X, Tan G, He C, Gao Y, Pan S, Jiang H, Zhang Y and Sun X: Hydrogen sulfide protects cardiomyocytes from myocardial ischemia-reperfusion injury by enhancing phosphorylation of apoptosis repressor with caspase recruitment domain. Tohoku J Exp Med 226: 275-285, 2012.

5. Thornberry NA and Lazebnik Y: Caspases: enemies within. Science 281: 1312-1316, 1998.

6. Canbay A, Taimr P, Torok N, Higuchi H, Friedman S and Gores GJ: Apoptotic body engulfment by a human stellate cell line is profibrogenic. Lab Invest 83: 655-663, 2003.
7. Mouw G, Zechel JL, Zhou Y, Lust WD, Selman WR and Ratcheson RA: Caspase-9 inhibition after focal cerebral ischemia improves outcome following reversible focal ischemia. Metab Brain Dis 17: 143-151, 2002.

8. Taylor RC, Cullen SP and Martin SJ: Apoptosis: controlled demolition at the cellular level. Nat Rev Mol Cell Biol 9: 231-241, 2008.

9. Srinivasula SM, Hegde R, Saleh A, Datta P, Shiozaki E, Chai J, Lee RA, Robbins PD, Fernandes-Alnemri T, Shi Y and Alnemri ES: A conserved XIAP-interaction motif in caspase-9 and Smac/DIABLO regulates caspase activity and apoptosis. Nature 410: 112-116, 2001.

10. Verhagen AM, Ekert PG, Pakusch M, Silke J, Connolly LM, Reid GE, Moritz RL, Simpson RJ and Vaux DL: Identification of DIABLO, a mammalian protein that promotes apoptosis by binding to and antagonizing IAP proteins. Cell 102: 43-53, 2000.

11. Dean EJ, Ranson M, Blackhall F and Dive C: X-linked inhibitor of apoptosis protein as a therapeutic target. Expert Opin Ther Targets 11: 1459-1471, 2007.

12. Vanden Berghe T, van Loo G, Saelens X, Van Gurp M, Brouckaert G, Kalai M, Declercq W and Vandenabeele P: Differential signaling to apoptotic and necrotic cell death by Fas-associated death domain protein FADD. J Biol Chem 279: 7925-7933, 2004

13. Wiessner C, Sauer D, Alaimo D and Allegrini PR: Protective effect of a caspase inhibitor in models for cerebral ischemia in vitro and in vivo. Cell Mol Biol (Noisy-le-grand) 46: 53-62, 2000.

14. Graeber MB, López-Redondo F, Ikoma E, Ishikawa M, Imai Y, Nakajima K, Kreutzberg GW and Kohsaka S: The microglia/macrophage response in the neonatal rat facial nucleus following axotomy. Brain Res 813: 241-253, 1998.

15. Lotocki G, Alonso OF, Frydel B, Dietrich WD and Keane RW: Monoubiquitination and cellular distribution of XIAP in neurons after traumatic brain injury. J Cereb Blood Flow Metab 23: 1129-1136, 2003.

16. Vellanki SH, Grabrucker A, Liebau S, Proepper C, Eramo A, Braun V, Boeckers T, Debatin KM and Fulda S: Small-molecule XIAP inhibitors enhance gamma-irradiation-induced apoptosis in glioblastoma. Neoplasia 11: 743-752, 2009.

17. Giagkousiklidis S, Vellanki SH, Debatin KM and Fulda S: Sensitization of pancreatic carcinoma cells for gamma-irradiation-induced apoptosis by XIAP inhibition. Oncogene 26: 7006-7016, 2007

18. Chen F, Xu C, Du L, Wang Y, Cao J, Fu Y, Guo Y, Liu Q and Fan F: Tat-SmacN7 induces radiosensitization in cancer cells through the activation of caspases and induction of apoptosis. Int J Oncol 42: 985-992, 2013.

19. Saito A, Hayashi T, Okuno S, Ferrand-Drake M and Chan PH: Interaction between XIAP and Smac/DIABLO in the mouse brain after transient focal cerebral ischemia. J Cereb Blood Flow Metab 23: 1010-1019, 2003.

20. Chai J, Shiozaki E, Srinivasula SM, Wu Q, Datta P, Alnemri ES and Shi Y: Structural basis of caspase-7 inhibition by XIAP. Cell 104: 769-780, 2001.

21. Riedl SJ, Renatus M, Schwarzenbacher R, Zhou Q, Sun C, Fesik SW, Liddington RC and Salvesen GS: Structural basis for the inhibition of caspase-3 by XIAP. Cell 104: 791-800, 2001.

22. Guijin H, QiYong G, XiaoDan Z, DaWei J, Xi G, ChunLai P, Liu W and XianWei D: Effect of 103Pd radioactive stent on caspase-9, cholangiocarcinoma cell growth and its radiosensitivity. Surg Oncol 20: 247-251, 2011.

23. Rudel T: Caspase inhibitors in prevention of apoptosis. Herz 24: 236-241, 1999.

24. Suzuki Y, Nakabayashi Y, Nakata K, Reed JC and Takahashi R: $\mathrm{X}$-linked inhibitor of apoptosis protein (XIAP) inhibits caspase-3 and -7 in distinct modes. J Biol Chem 276: 27058-27063, 2001.

25. Stahl JS and Thumser ZC: Dynamics of abducens nucleus neurons in the awake mouse. J Neurophysiol 108: 2509-2523, 2012.

26. van Loo G, Saelens X, van Gurp M, MacFarlane M, Martin SJ and Vandenabeele P: The role of mitochondrial factors in apoptosis: a Russian roulette with more than one bullet. Cell Death Differ 9: 1031-1042, 2002. 